\title{
Post-COVID Reactive Arthritis
}

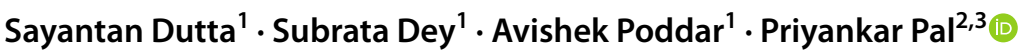

Received: 13 June 2021 / Accepted: 12 October 2021 / Published online: 23 October 2021

(c) Dr. K C Chaudhuri Foundation 2021

To the Editor: A 14-y-old male presented with acute onset polyarthritis (right elbow, bilateral knees, and ankles) for $4 \mathrm{~d}$. There were no associated symptoms and the pain was unresponsive to naproxen. He had history of mild COVID (RT-PCR positive) $3 \mathrm{wk}$ back together with the other family members and they had been managed conservatively at home. His father also had similar post-COVID knee arthritis that required hospitalization.

Investigations showed the total leukocyte count of 5700/ $\mathrm{cmm}$, neutrophils $54 \%$, lymphocytes $42 \%$, CRP - $4 \mathrm{mg} / \mathrm{L}$, and ESR - 15. COVID IgG was positive (3.93, normal $<1$ ). HIV and mycoplasma serology, ASO, ANA, anti-CCP, HLA-B27 were negative. There was no antecedent history of gastrointestinal or urinary tract infection, so we did not investigate for reactive arthritis secondary to other arthritogenic organisms. Ultrasonography showed effusion in both knee joints, and no tenosynovitis. Since he refused intraarticular triamcinolone and the pain was unresponsive to naproxen, he was initiated on IV methylprednisolone at $2 \mathrm{mg} / \mathrm{kg} / \mathrm{d}$. This resulted in significant improvement and was continued for $3 \mathrm{~d}$. He was discharged on oral prednisolone that was tapered off over $15 \mathrm{~d}$. He remains asymptomatic on follow-up.

With the ongoing pandemic, a myriad of COVIDassociated inflammatory presentations are being reported. Although a few cases of post-COVID reactive arthritis have been reported in adults [1, 2], this is rarely described in children [3]. Molecular mimicry has been proposed [4] as the possible immunological mechanism. Most of the reported arthritis occurred 2 to 4 wk after detection of COVID positivity, predominantly affecting the knees and ankles, but also, occasionally, the small joints of the hands. This is a seronegative arthritis, with normal blood counts and inflammatory markers. The reported modalities of treatment vary from intra-articular steroids to COX2 inhibitors with a favorable response, without any residual damage or erosion.

\section{Declarations}

Conflict of Interest None.

\section{References}

1. Jali I. Reactive arthritis after COVID-19 infection. Cureus. 2020;12:e11761.

2. Baimukhamedov C, Barskova T, Matucci-Cerinic M. Arthritis after SARS-CoV-2 infection. Lancet Rheumatol. 2021;3:e324-5.

3. Sinaei R, Pezeshki S, Parvaresh S, et al. Post SARS-CoV-2 infection reactive arthritis: a brief report of two pediatric cases. Pediatr Rheumatol Online J. 2021;19:89.

4. Gasparotto M, Framba V, Piovella C, Doria A, Iaccarino L. PostCOVID-19 arthritis: a case report and literature review. Clin Rheumatol. 2021;40:3357-62.

Publisher's Note Springer Nature remains neutral with regard to jurisdictional claims in published maps and institutional affiliations.
Priyankar $\mathrm{Pal}$

1 Department of Pediatrics, Apollo Gleneagles Hospital, Kolkata, West Bengal, India

2 Department of Pediatric Rheumatology, Institute of Child Health, Kolkata, West Bengal 700017, India

3 Department of Pediatric Rheumatology, Apollo Gleneagles Hospital, Kolkata, West Bengal, India 